\title{
Stochastic resonance and diffusion in periodic potentials
}

\author{
J Kallunki†, M Dubé $\dagger$ and T Ala-Nissila $† \ddagger$ \\ $\dagger$ Helsinki Institute of Physics and Laboratory of Physics, PO Box 1100, FIN-02150 HUT, Espoo, \\ Finland \\ \$ Department of Physics, Brown University, Providence, RI 02912-1843, USA
}

Received 18 May 1999

\begin{abstract}
We study the diffusive motion of a particle in a periodic adiabatic potential under the influence of a time-periodic bias, concentrating on the case of high friction and small bias with respect to the potential barriers. We find that the distribution of escape times shows a series of peaks, whose amplitude varies with temperature, in analogy to stochastic resonance in standard double-well systems. However, the hydrodynamic diffusion coefficient does not show a stochastic resonance type of maximum.
\end{abstract}

\section{Introduction}

The concept of stochastic resonance (SR), introduced more than ten years ago [1,2], has found a broad range of applications in many physical two-well systems, such as Josephson junctions or laser rings, as well as in more complex systems, such as neural networks, or extended systems showing spatiotemporal chaos (see e.g. reference [2] for an extensive review). Some attempts have also been made to apply these concepts to multi-state systems, focusing mostly on diffusive particle dynamics in periodic potentials [3-6].

A stochastic system subject to a time-periodic perturbation often shows an enhancement of its transport properties. The term SR then refers to the situation where this enhancement becomes maximal for some value of the stochastic noise. For a simple two-well system, stochastic resonance may be said to occur when there is optimal matching between the natural timescale of the unperturbed system and the period of the oscillating perturbations [7], i.e.,

$$
T_{k}=\pi \Omega^{-1}
$$

where $T_{k}$ is the average escape time of the system (such that $T_{k}^{-1}$ is the rate of transition between the wells) and $\Omega$ is the frequency of the oscillating perturbation. At this point, the noise-induced hopping occurs statistically in phase with the bias (see reference [8] for a more detailed discussion about this point).

Mathematically, the SR problem can often be described by a stochastic differential equation of the Langevin type, with a time-periodic perturbation term added [2]. Such Langevin equations also describe the diffusive motion of atoms, the classical example of this being Brownian motion. A particularly interesting special case of such diffusive processes is the diffusion of adatoms on surfaces, where there is an underlying periodic potential [9]. Surface diffusion plays an important role in many problems, such as catalysis or surface growth [10]. It would thus be of great interest to apply the concept of SR to surface diffusion. It is well known that when a DC electric field is applied to diffusing adatoms, electromigration [11] takes place 
and strongly influences the morphology and time evolution of metallic and semiconductor surfaces.

In fact, several studies of SR in periodic potentials have already been performed. For a static bias and low friction [3], it has been shown that the adatom mobility can show a maximum as a function of noise intensity. For an oscillating bias, recent studies [4-6] have indicated that the diffusion coefficient can be significantly enhanced, in a way similar to SR. These studies were, however, mostly conducted in the limit where the energy associated with the amplitude of the bias is larger than the height of the diffusion barrier, a regime that unfortunately may be experimentally unrealistic for typical adatom diffusion on surfaces. Also, in this limit the effect of the confining periodic potential is mostly lost, and in this sense the situation it is not the same as in the true SR phenomenon that occurs for a double-well potential when the perturbation amplitude is much smaller than the barrier.

Since in the high-friction limit of diffusion in a periodic potential the adatom motion consists of activated transitions between the nearest potential minima [9], one would expect a matching condition similar to equation (1) to hold even for small-amplitude perturbations. The relevant question then concerns the existence of SR in the hydrodynamic transport coefficients, such as the (tracer) diffusion coefficient $D_{T}[9,12]$. This is the question examined in the present paper. We perform a detailed study of diffusive motion under an oscillating bias, allowing us to identify which physical quantities show 'SR-like' features, and which do not. The present study is concentrated on the high-friction regime.

The organization of this paper is as follows. In section 2, we describe our model and the physical observables associated with it. In section 3, we present the results of our simulations for the spatial correlation function, supplemented by a theoretical analysis based on a master equation. The main result is that the diffusion coefficient is a monotonically increasing function of temperature, showing no signs of SR. In section 4, we show that the most pronounced effects of the oscillating bias are found in the distribution of escape times [8]. This distribution, divided into a distribution for 'parallel' and 'anti-parallel' transitions, is strongly peaked at times related to the period of the oscillating driving force, and the amplitude of a given peak goes through a maximum when a matching condition between $\Omega$ and $T_{k}$ is established. Nevertheless, this has no discernible influence on the diffusive motion in the hydrodynamic limit, since the total escape time distribution remains a pure exponential. We conclude with possible applications of these ideas in section 5 .

\section{Diffusion in periodic potentials}

The 1D motion of a Brownian particle in a periodic potential with a time-periodic bias is modelled by the Langevin equation

$$
M \frac{\mathrm{d}^{2} x}{\mathrm{~d}^{2} t}+M \gamma \frac{\mathrm{d} x}{\mathrm{~d} t}+\frac{2 \pi}{a} V_{0} \sin \left(\frac{2 \pi x}{a}\right)=F_{a} \cos (\omega t+\phi)+\Gamma(t)
$$

where $\Gamma$ is Gaussian white noise, with autocorrelations

$$
\left\langle\Gamma(t) \Gamma\left(t^{\prime}\right)\right\rangle=2 k_{B} T M \gamma \delta\left(t-t^{\prime}\right) .
$$

Here $T$ is the temperature and $\gamma$, the friction coefficient, arises microscopically from the interaction of the adatom with electrons or phonons [9]. The bias force $F_{a}$ gives rise to an energy change $E_{a}=a F_{a}$ across one lattice spacing.

The relevant timescales of the problem are the dissipation time, $\gamma^{-1}$, the vibrational timescale, $\omega_{0}^{-1}=a\left(M / V_{0}\right)^{1 / 2} / 2 \pi$ and the period of the external forcing $T_{\omega}=2 \pi \omega^{-1}$. The phase of the driving force is denoted by $\phi$. The ratio $\Omega_{0}=\omega_{0} / \gamma$ determines the high- $\left(\Omega_{0} \ll 1\right)$ 
and low-friction $\left(\Omega_{0} \gg 1\right)$ regimes. In this study, we only consider $\Omega_{0} \ll 1$, with the ratio $\omega_{0} / \omega$ arbitrary.

A main quantity of interest in this case is the correlation function

$$
\tilde{C}\left(t, t_{0} ; \phi_{0}\right)=\left\langle\left(x\left(t+t_{0}\right)-x\left(t_{0}\right)\right)^{2}\right\rangle
$$

which is not time-translationally invariant, due to the periodic bias, but depends on both time arguments $t, t_{0}$, through the initial phase $\phi_{0}=\Omega \tau_{0}+\phi$. In realistic experimental situations, this function must be averaged over $\phi_{0}$, using an uniform distribution with limits $0 \leqslant \phi_{0} \leqslant 2 \pi[14]$. Equation (4) then reduces to a time-translationally invariant form $\tilde{C}(t)$ :

$$
\tilde{C}(t)=\frac{1}{2 \pi} \int_{0}^{2 \pi} \tilde{C}\left(t, t_{0} ; \phi_{0}\right) \mathrm{d} \phi_{0} .
$$

The tracer diffusion coefficient of the particle (adatom) $D_{T}$ is obtained in the limit $t \rightarrow \infty$ :

$$
D_{T}=\lim _{t \rightarrow \infty} \frac{1}{2 t} \tilde{C}(t)
$$

In the absence of periodic forcing $\left(E_{a}=0\right)$, the high-temperature $\left(k_{B} T \gg V_{0}\right)$ limit of the diffusion coefficient is that of a free Brownian particle, $D_{T}=k_{B} T / M \gamma$, while at low temperatures and in the high-friction limit, it may be approximated by $D_{T}=a^{2} T_{k}^{-1}[9]$.

Another quantity of interest is the escape time distribution $\rho(\tau)$, defined as the probability density that a transition occurs at a time $\tau$ after the initial arrival of the particle [8]. To account for the periodic nature of the potential, two different types of transition are defined. A parallel transition at time $t_{i}+1$ occurs if the particle occupies the position $x$ at time $t_{i}$, while it was previously at position $x \mp a$, with the subsequent transition (at time $t_{i+1}$ ) taking the particle to position $x \pm a$. The motion is thus continuous for at least two consecutive transitions and the statistical properties of the time interval $\tau=t_{1+1}-t_{i}$ are determined by the distribution $\rho_{\|}(\tau)$. On the other hand, if the transition at time $t_{i+}$ takes the particles to position $x \mp a$, such that the motion is reversed, then the transition is termed anti-parallel and is described by the distribution $\rho_{\perp}(\tau)$.

In the absence of any bias, both $\rho_{\|}$and $\rho_{\perp}$ are simple exponential, and the total escape time distribution $\rho(\tau)=\rho_{\|}(\tau)+\rho_{\perp}(\tau)$ is related to the mean escape time $T_{k}$ as

$$
\rho(\tau)=\frac{1}{T_{k}} \mathrm{e}^{-\tau / T_{k}}
$$

In cases of high and intermediate friction, and in the low-temperature limit $k_{B} T \ll V_{0}$, it is sufficient to use Kramers' result [13]

$$
T_{k}^{-1}=r_{k}(T)=\frac{\omega_{0}^{2}}{2 \pi M \gamma} \mathrm{e}^{-2 V_{0} / k_{B} T} .
$$

\subsection{Numerical methods}

The 1D Brownian particle is numerically simulated by using the dimensionless space and time variables $q=2 \pi x / a$ and $\tau=\gamma t$. The Langevin equation then becomes

$$
\frac{\mathrm{d}^{2} q}{\mathrm{~d}^{2} \tau}+\frac{\mathrm{d} q}{\mathrm{~d} \tau}+\Omega_{0}^{2} \sin (x)=E_{0} \Omega_{0}^{2} \cos \left(\tilde{\Omega} \tau+\phi_{0}\right)+\epsilon(\tau)
$$

with the dimensionless bias $E_{0}=E_{a} / 2 \pi V_{0}$, driving frequency $\Omega=\omega / \gamma, \Omega_{0}=\omega_{0} / \gamma$ and the noise correlated as

$$
\left\langle\epsilon(\tau) \epsilon\left(\tau^{\prime}\right)\right\rangle=\frac{2 k_{B} T}{V_{0}} \Omega_{0}^{2} \delta\left(\tau-\tau^{\prime}\right) .
$$


The reduced equation (9) is then integrated by means of the standard Ermarck algorithm [16]. The dimensionless correlation function is $C(\tau)=(2 \pi / a)^{2} \tilde{C}(\gamma t)$ and the diffusion coefficient $D=(2 \pi / a)^{2} D_{T} / \gamma$. In these units, the Kramers rate

$$
R_{k}=\left(\Omega_{0}^{2} / 2 \pi\right) \exp \left(-2 V_{0} / k_{B} T\right) .
$$

\section{The correlation function and tracer diffusion}

In this section, we concentrate on the behaviour of the mean square displacement in the presence of an oscillating bias. Our main conclusion is that, although the diffusion coefficient is enhanced by the oscillating bias, it shows none of the features usually associated with stochastic resonance. We emphasize again that we are only considering the high-friction regime here.

\subsection{Numerical results}

Figure 1 shows the phase-averaged correlation function $C(\tau)$ in the high-friction regime. As expected, it is composed of a linear part upon which an oscillatory part in superimposed. The diffusion coefficient, defined by the slope of the linear component, is shown in figure 2 . It is a monotonically increasing function of temperature, showing no maxima. We note that the range of temperatures used is such that it sweeps through the double-well matching condition $T_{\Omega}=2 R_{k}^{-1}(T)$. The amplitude of the oscillations in $C(\tau)$, shown on the inset of figure 2 , also increases monotonically as a function of temperature, and saturates to a constant value.

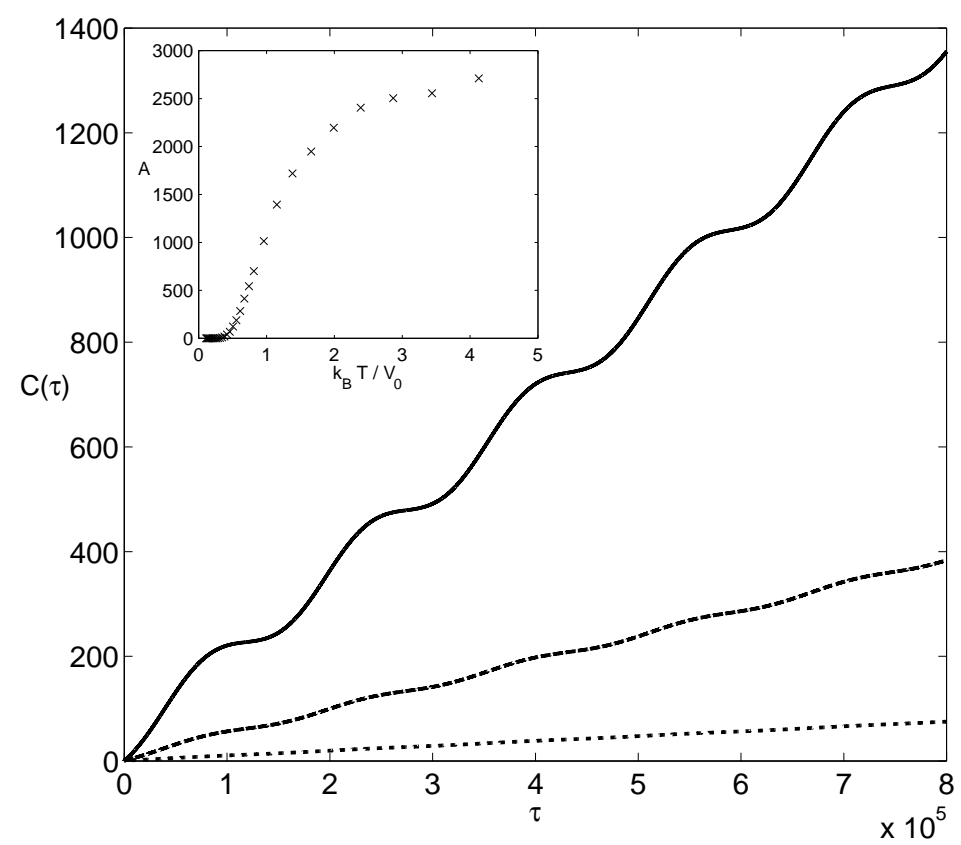

Figure 1. The mean square displacement of the particle as a function of time for various temperatures, with $\Omega_{0}=0.1, \Omega=4 \times 10^{-5} \gamma, E_{0}=0.15$ and $k_{B} T / V_{0}=0.26,0.35$ and 0.46 (from bottom to top). In the inset, we show the amplitude $A$ of the oscillations in $C(\tau)$ as a function of temperature, up to $k_{B} T / V_{0} \simeq 4$. 


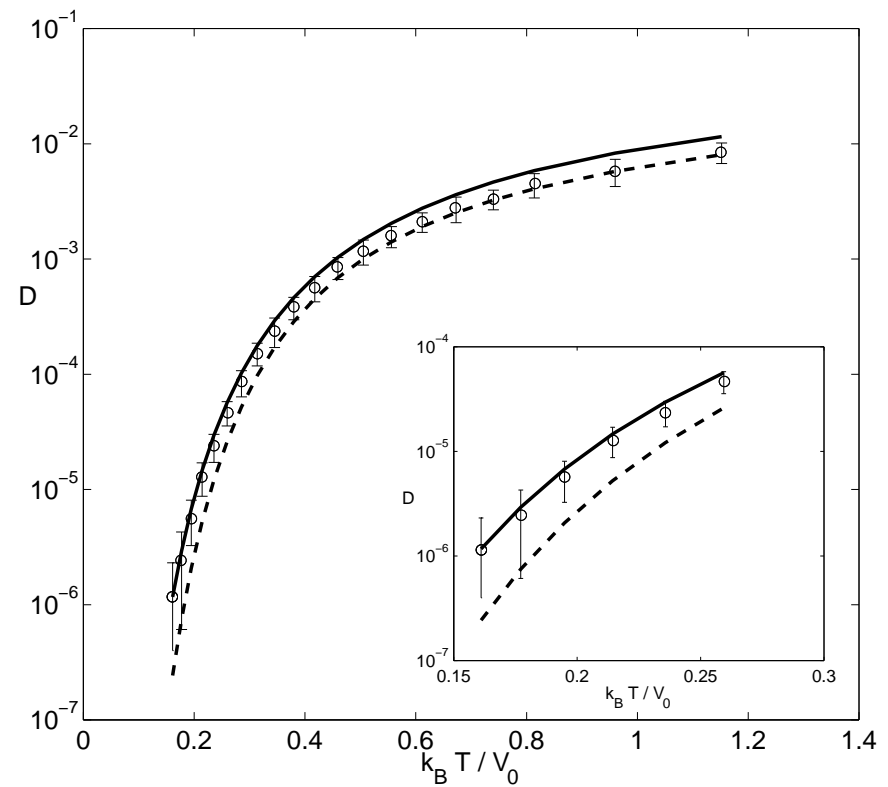

Figure 2. The diffusion coefficient $D(T)$ as a function of temperature for $\Omega=4 \times 10^{-5} \gamma$ and $E_{0}=0.15$. We show the numerical results (open circles), the theoretical results (full line), based on equation (6), and the value of $D(T)$ if $E_{a}=0$ (dashed line).

We emphasize that these results occur in the limit $E_{a} \ll V_{0}$. It is possible to observe features similar to those of SR in the behaviour of $D$ in the inverse limit $E_{a} \gg V_{0}$, which is not considered here for the reasons discussed in the introduction.

\subsection{Analytical results}

We now show that the qualitative behaviour of our results can be recovered by considering a simple theoretical model. In the high-friction regime, the diffusion process can be analysed in terms of the Master equation

$$
\frac{\mathrm{d} P_{n}(\tau)}{\mathrm{d} \tau}=-\left[W_{+}(\tau)+W_{-}(\tau)\right] P_{n}(\tau)+P_{n+1}(\tau) W_{-}(\tau)+P_{n-1}(\tau) W_{+}(\tau)
$$

where $P_{n}(\tau)$ is the probability that the Brownian particle is in the $n$th potential well at time $\tau$. The time-dependent transition rates $W_{ \pm}(\tau)$ are taken to be of the Kramers form [13]:

$$
W_{ \pm}(\tau ; \phi)=\frac{\Omega_{0}^{2}}{2 \pi} \exp \left(-\frac{2 V_{0}}{k_{B} T} \pm \frac{E_{a}}{2 k_{B} T} \sin (\Omega \tau+\phi)\right) .
$$

The formal solution of the master equation can be expressed in terms of the integral $\alpha_{ \pm}\left(\tau, \tau_{0} ; \phi\right)$ over the transition rates:

$$
\begin{aligned}
\alpha_{ \pm}\left(\tau, \tau_{0} ; \phi\right) & =\int_{\tau_{0}}^{\tau+\tau_{0}} \mathrm{~d} \tau^{\prime} W_{ \pm}\left(\tau^{\prime} ; \phi\right) \\
& =R_{k}(T) I_{0}\left(E_{a} / 2 k_{B} T\right)\left(\Delta \tau^{*}\right)+R_{k}(T) \int_{\Delta \tau^{*}}^{\tau+\tau_{0}} \mathrm{~d} \tau^{\prime} \exp \left( \pm \frac{E_{a}}{2 k_{B} T} \sin \left(\Omega \tau^{\prime}+\phi\right)\right)
\end{aligned}
$$


where $I_{0}(u)$ is the modified Bessel function and $\Delta \tau^{*}=2 \pi N / \Omega, N$ being the total number of periods between times $\tau_{0}$ and $\tau$. In the limit of large $\tau$, the value of

$$
\alpha\left(\tau, \tau_{0} ; \phi_{0}\right)=\tau R_{k}(T) I_{0}\left(E_{a} / 2 k_{B} T\right)
$$

for all values of $E_{a} / 2 k_{B} T$.

The probability $P_{n}(t)$, with initial condition $P_{n}(0)=\delta_{n, n_{0}}$, has the solution

$$
P_{n}(\tau)=\mathrm{e}^{-\left(\alpha_{+}+\alpha_{-}\right)}\left(\frac{\alpha_{+}}{\alpha_{-}}\right)^{\left(n-n_{0}\right) / 2} I_{n-n_{0}}\left(2 \sqrt{\alpha_{+} \alpha_{-}}\right)
$$

and the correlation function, for fixed phase, is

$$
C\left(\tau, \tau_{0} ; \phi\right)=\left[\alpha_{+}-\alpha_{-}\right]^{2}+\alpha_{+}+\alpha_{-} .
$$

A perturbative result can by obtained by expanding the last term of equation (13) in powers of $E_{a} / 2 k_{B} T$ followed by an averaging over the initial phase, which results in

$$
C(\tau)=2 R_{k}(T) I_{0}\left(E_{a} / 2 k_{B} T\right) \tau+R_{k}^{2}(T)\left(\frac{E_{a}}{k_{B} T \Omega}\right)^{2}(1-\cos (\Omega \tau)) .
$$

The diffusion coefficient $D=D(T)$ is obtained from the definition of equation (6) as

$$
D=R_{k}(T) I_{0}\left(E_{a} / 2 k_{B} T\right)
$$

which is a result valid in the limits $E_{a} \ll V_{0}$ and $k_{B} T \ll V_{0}$, but for all values of $E_{a} / 2 k_{B} T$. It clearly shows the monotonic increase of $D(T)$, and compares quite well with our numerical results. Deviations occur when $k_{B} T \sim V_{0}$, outside the range of validity of Kramers' results.

For $E_{a} \ll 2 k_{B} T$, the diffusion coefficient can be approximated by

$$
D(T) \approx D\left(E_{a}=0\right)\left(1+\left(\frac{E_{a}}{4 k_{B} T}\right)^{2}\right)
$$

which is different from what is found in reference [5]. We note that this simple limit predicts a maximum in the enhancement of the diffusion coefficient at a temperature $k_{B} T \sim V_{0}$, a limit out of the range of validity of the analysis. In the limit $E_{a} / k_{B} T \gg 1$, the diffusion coefficient becomes

$$
D(T) \approx R_{k}(T)\left(\frac{k_{B} T}{2 \pi E_{a}}\right)^{1 / 2} \mathrm{e}^{E_{a} / k_{B} T} .
$$

The result for $D(T)$ can be intuitively understood by an averaging of the Kramers rate over the period of the oscillating bias $T_{\Omega}$, yielding the effective transition rate

$$
r_{\text {eff }}=\frac{\Omega_{0}^{2}}{2 \pi} \frac{1}{T_{\Omega}} \int_{0}^{T_{\Omega}} \exp \left(-\frac{2 V_{0}}{k_{B} T} \pm \frac{E_{a}}{k_{B} T} \sin (\Omega \tau)\right) \mathrm{d} \tau=R_{k} I_{0}\left(\frac{E_{a}}{k_{B} T}\right)
$$

which is similar to equation (17), since $D(T) \sim r_{\text {eff }}$.

In the limit $E_{a} \ll k_{B} T$, the amplitude of the oscillations in $C(\tau)$, found from equation (16), shows a maximum at the temperature $k_{B} T=2 V_{0}$, a feature also associated with SR but again far beyond the validity of the approximations. Such a result is not physically valid. Instead, the amplitude saturates at a plateau value, which may be obtained from the hightemperature limit, $k_{B} T \gg V_{0}$, of equation (9). Neglecting the spatial potential $V(x)$, it is straightforward to obtain the phase-averaged correlation function as

$$
C(\tau)=2 \frac{k_{B} T}{V_{0}} \Omega_{0}^{2} \tau+\frac{E_{a}^{2} \Omega_{0}^{4}}{\Omega^{2}}[1-\cos (\Omega \tau)]
$$

yielding the plateau value, and the diffusion coefficient $D(T)=2 k_{B} T \Omega_{0}^{2} / V_{0}$, such that $D_{T}=k_{B} T /(M \gamma)$, valid for a free Brownian particle. 


\section{Escape time distributions}

The escape time distributions may be used to study the synchronization between jumps, in a way similar to the two-well case [2]. Both quantities can also be measured experimentally, through STM measurements [15]. The escape time distributions for parallel $\left(\rho_{\|}(\tau)\right)$ and antiparallel $\left(\left(\rho_{\perp}(\tau)\right)\right.$ transitions, shown in figure 3 , present some interesting characteristics arising from the driving of period $T_{\Omega}=2 \pi \Omega^{-1}$. In both cases, the distributions show pronounced peaks at times $\tau_{\max , n}^{\|}=n T_{\Omega}$ for parallel transitions, and at $\tau_{\max , n}^{\perp}=(n+1) T_{\Omega} / 2$ for antiparallel ones. Although both $\rho_{\|}$and $\rho_{\perp}$ are peaked functions of time, the total escape time distribution

$$
\rho(\tau)=\rho_{\|}(\tau)+\rho_{\perp}(\tau)
$$

still shows purely exponential behaviour as

$$
\rho(\tau)=\frac{1}{2 T_{k}} \mathrm{e}^{-\tau / T_{k}}
$$

as also shown in figure 3.

The peaks in $\rho_{\|}$and $\rho_{\perp}$ are obviously related to the enhanced transition opportunities for parallel transitions after an integer multiple of the driving period. Likewise, anti-parallel
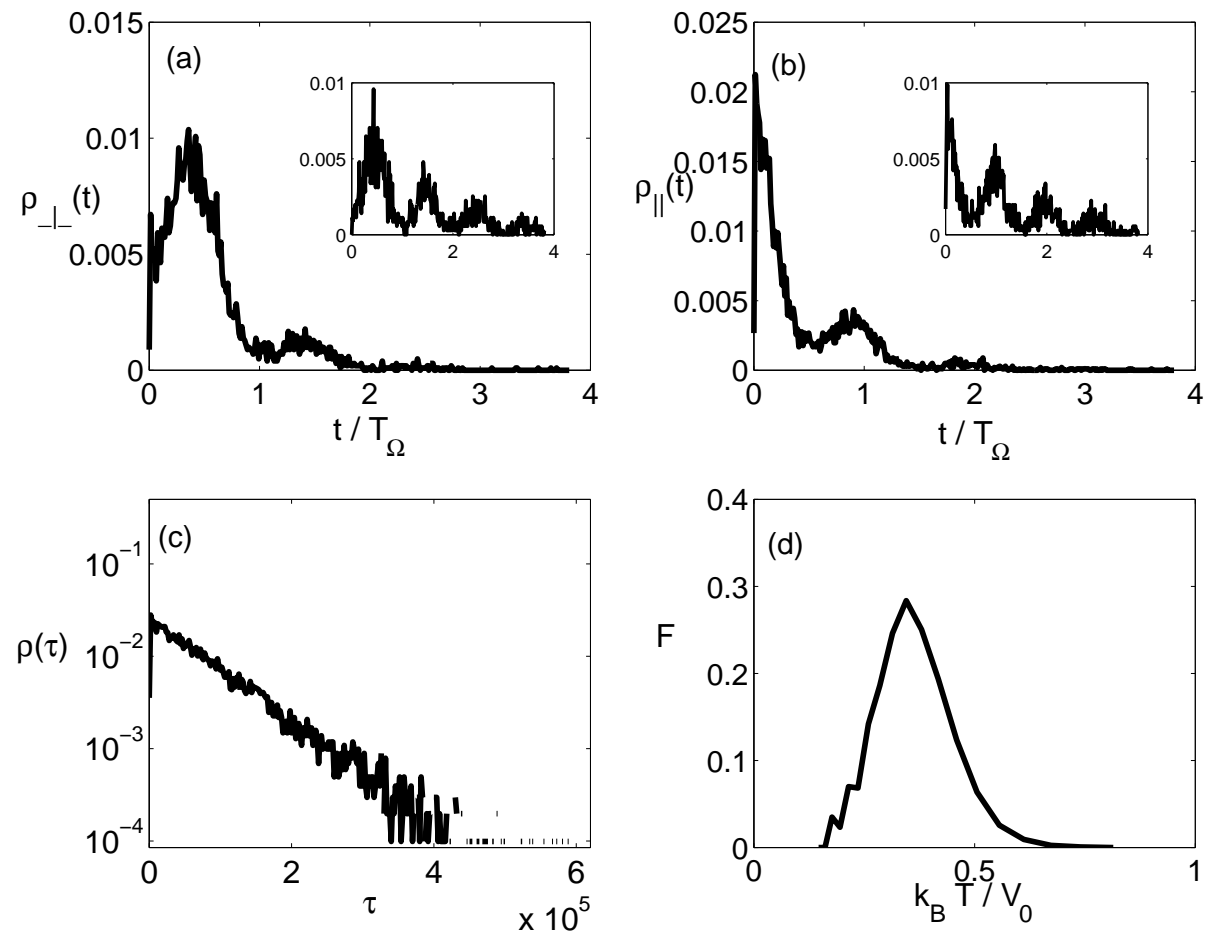

Figure 3. Escape time distributions with parameter $\Omega=4 \times 10^{-5}$. (a) The distribution for parallel transitions, at temperature $k_{B} T / V_{0}=0.35$, showing the peaks at integer values of the driving period. In the inset, we show the same quantity, but at temperature $k_{B} T / V_{0}=0.29$. (b) The distribution for parallel transitions with identical parameters, showing the peak at halfinteger values of the driving period. (c) The total escape time distribution $\rho(\tau)$ at temperature $k_{B} T / V_{0}=0.35$, showing pure exponential behaviour. (d) The amplitude of the first peak in $\rho_{\|}$as a function of temperature. 
transitions are favoured after a half-integer number of periods. To further investigate this behaviour, the amplitude $F(T)$ of the first peak in the anti-parallel distribution $\rho_{\perp}(t)$, is defined following [7] as

$$
F=\int_{T_{\Omega} / 4}^{3 T_{\Omega} / 4} \rho_{a}(t) \mathrm{d} t .
$$

This function shows a clear maximum as a function of temperature, a feature of SR also seen in bistable systems [2,7]. As in this case, the maximum corresponds to an optimal synchronization between the driving period and the average escape time $T_{k}(T)$. The maximum occurs at the matching condition

$$
2 T_{k}(T)=T_{\Omega}
$$

Using our data, we estimate $T_{k}=8 \times 10^{4}$, which corresponds nicely to the value $T_{\Omega}=1.57 \times 10^{5}$ chosen in the simulations. If the Kramers rates can be used, the maximum in the distribution then occurs at a temperature $k_{B} T_{\max } / 2 V_{0}=1 / \ln \left(\omega_{0}^{2} / \gamma \omega\right)$. We emphasize, however, that this maximum does not result in a peak structure for the diffusion coefficient $D(T)$, and is even less likely to result in some form of either 'ballistic' or 'localized' motion. Although $\rho_{\|}$and $\rho_{\perp}$ may have peaks at different temperatures, their sum is always of pure exponential form, with mean escape time $T_{k}$, implying purely diffusive behaviour. Making the distinction between $\rho_{\|}$and $\rho_{\perp}$ is the only experimentally relevant way to study stochastic resonance in periodic systems. These concepts might also be useful for surfaces with broken symmetry (due to, e.g., steps and terraces [17]) and for interacting systems [9].

\section{Conclusions and discussion}

The results presented above show that no peaks in the temperature behaviour of the diffusion coefficient result from the application of an oscillating small bias. Features associated with SR are seen in the escape time distributions, but not in the hydrodynamic transport coefficients. The main effect of the oscillating bias in the limit $E_{a} \ll V_{0}$ is an enhancement of the diffusion coefficient. This enhancement is the of order of $E_{a} / V_{0}$ if $E_{0} \ll k_{B} T$, or $\exp \left(E_{a} / k_{B} T\right)$ if $E_{a} \gg k_{B} T$, always assuming $E_{a}$ and $k_{B} T$ to be much smaller than the barrier height $V_{0}$.

Several problems, however, remain to be solved. Electromigration [11] is usually an extremely small effect, causing changes in the potential barrier $E_{a} / V_{0}$ of less than $1 \%$ (although values as high as $E_{a} / V_{0} \approx 0.1$ have been quoted in reference [17]). These very small values mean that no discernible effects on the diffusion coefficient will occur, at least in the highfriction limit, unless temperature is very low.

Nevertheless, two other options deserve further study. The low-friction limit, characterized by long jumps between the wells, may well present a greater sensibility to the oscillating field, as already hinted in reference [3]. Furthermore, the effect of an oscillating bias on diffusion on stepped terraces [17] has been studied in the limit $\Omega \rightarrow 0$. Since the DC limit is difficult to obtain experimentally, it is imperative that these studies be extended to the high-frequency case. Work in this direction is already in progress.

\section{Acknowledgments}

We thank S Majaniemi, E Hernandez-García and P Colet for useful discussions at the early stages of this work. This work was supported by the Academy of Finland. 


\section{References}

[1] McNamara B and Wiesnefeld K 1989 Phys. Rev. A 394854

[2] Gammaitoni L, Hänggi P, Jung P and Marchesoni F 1998 Rev. Mod. Phys. 70223

[3] Marchesoni F 1997 Phys. Lett. A 23161

[4] Gang H, Daffertshofer A and Haken H 1996 Phys. Rev. Lett. 764874

[5] Kim Y W and Sung W 1998 Phys. Rev. E 576237

[6] Hernandez-García E, Ala-Nissila T, Colet P, Dubé M and Majaniemi S 1998 Ann. Fis. Mono. RSEF 10

[7] Gammaitoni L, Marchesoni F, Menichella-Saetta E and Santucci S 1989 Phys. Rev. Lett. 62349

[8] Choi M H, Fox R F and Jung P 1998 Phys. Rev. E 576335

[9] Ala-Nissila T and Ying S C 1992 Prog. Surf. Sci. 39227

[10] Barabási A-L and Stanley H E 1995 Fractal Concepts in Surface Growth (Cambridge: Cambridge University Press)

[11] Yasunaga H and Natori A 1992 Surf. Sci. Rep. 15205

Rous P J, Einstein T L and Williams E D 1994 Surf. Sci. 315 L995

[12] Gomer R 1990 Prog. Surf. Sci. 53917

[13] Hänggi P, Talkner P and Borkovec M 1990 Rev. Mod. Phys. 62251

[14] Jung P 1993 Phys. Rep. 234175

[15] Swartzentruber B S 1996 Phys. Rev. Lett. 76459

[16] Allen M P and Tildesley D J 1994 Computer Simulation of Liquids (Oxford: Clarendon)

[17] Derényi I, Lee C and Barabási A-L 1998 Phys. Rev. Lett. 801473 